\section{Toeplitz Operators}

\section{G.J. Murphy}

There are few classes of operators on a Hilbert space about which one has very detailed information, apart from the normal operators and the compact operators. An exceptional class about which much is known is the class of Toeplitz operators. This paper gives a brief survey of some aspects of their theory, from its origin near the begining of this century to the present day.

\section{The basic results}

The study of Toeplitz operators was initiated in a paper in 1911 (by Toeplitz [13]) in which the relationship of finite square matrices that are constant on diagonals to the corresponding infinite matrices was investigated. The class of Wiener-Hopf operators was studied in parallel (from 1931) until Rosenblum [11] observed in 1965 that that the two classes of operators are unitarily equivalent. There now exists a vast literature on this area. The theory is interesting in its own right, but also has applications to and connections with many other areas, for example, Function. Theory, Prediction Theory, $\mathrm{C}^{*}$-algebras, other areas of Operator Theory, Probability, and Physics.

Having indicated why Toeplitz operators are studied, let us now define them and look at some of their properties.

Let $\mathrm{T}$ denote the circle group, $\mathrm{T}=\{z \in \mathrm{C}|| z \mid=1\}$, and let $\lambda$ denote normalized Haar measure (= normalized arc length) on $\mathbb{T}$. For $p \in[1,+\infty]$ let $L^{p}=L^{p}(T, \lambda)$. If $\varphi \in L^{\infty}$ we get a bounded linear operator $M_{\varphi}$ on $L^{2}$ by setting

$$
M_{\varphi}(f)=\varphi f \quad\left(f \in L^{2}\right) .
$$

$M_{\varphi}$ is called the Laurent operator with symbol $\varphi$. It is of course a normal operator, i.e. it commutes with its adjoint, and the $\operatorname{map} \varphi \mapsto M_{\varphi}$ is an isometric *-homomorphism of $L^{\infty}$ into $B\left(L^{2}\right)$ (for any Hilbert space $H$, we let $B(H)$ denote the Banach algebra of all bounded linear operators on $H$ ). The matrices of these operators are very special when taken relative to the standard orthonormal basis $\left(e_{n}\right)_{n \in \mathbb{Z}}$ (where $e_{n}: z \mapsto z^{n}$ ) - they are constant

along diagonals. However our interest is not really in Laurent operators, whose theory is very easy, but in their compressions to a certain subspace $B^{3}$. Recall that for $p \in[1,+\infty]$ the $H a r d y$ space $H^{p}$ is the set of all $f \in L^{p}$ with "negativen Fourier coefficients $\left\langle f, e_{n}\right\rangle=\int f \bar{e}_{n} d \lambda(n<0)$ all equal to zero. Let $P$ denote the projection of $L^{2}$ onto $H^{2}$. If $\varphi \in L^{\infty}$ then $T_{\varphi} \in B\left(H^{2}\right)$ is defined by setting

$$
T_{\varphi}(f)=P(\varphi f) \quad\left(f \in H^{2}\right) .
$$

$T_{\varphi}$ is called the Toeplitz operator with symbol $\varphi$. It might appear at first sight that the theory of Toeplitz operators should be like that of Laurent operators, since the difference between their definitions may appear trivial, but in fact the two theories are profoundly different. The theory of Toeplitz operators is deep, and hard.

Let us begin by noting some elementary facts. Every Toeplitz operator has matrix with constant diagonals relative to the standard orthonormal basis $\left(e_{n}\right)_{n=0}^{\infty}$, and conversely any $T \in B\left(B^{2}\right)$ with such a matrix relative to $\left(e_{n}\right)_{n=0}^{\infty}$ is a Toeplitz operator. One has $\left\|T_{\varphi}\right\|=r\left(T_{\varphi}\right)$ (the spectral radius) $=\|\varphi\|_{\infty}$. The spectral theory of $T_{\varphi}$ is complicated by the fact that invertibility of $T_{\varphi}$ is not equiva lent to inyertibility of $\varphi$ (although the corresponding statement is true for $\left.M_{\varphi}\right)$. One does have implication in one direction however: if $T_{\varphi}$ is invertible then $\varphi$ is invertible. Hence $\sigma(\varphi) \subseteq \sigma\left(T_{\varphi}\right)$, a result due to Hartman and Wintner [6]. $\sigma()$ denotes the spectrum - for $a$ an element of an algebra having a unit 1 the spectrum $\sigma(a)$ of $a$ is the set of all $z \in \mathrm{C}$ such that $z 1-a$ is not invertible. As indicated above one does not in general have $\sigma\left(T_{\varphi}\right)=\sigma(\varphi)$. An example is provided by the unilateral shift $U=T_{e_{2}}$ as its spectrum is the closed unit disc, but the spectrum of $e_{1}$ is the unit circle. However $\sigma\left(T_{\varphi}\right)$ is not too much bigger than $\sigma(\varphi)$, because $\sigma\left(T_{\varphi}\right)$ is contained in the closed convex hull of $\sigma(\varphi)$ (Brown-Halmos [1]).

The above results are relatively near the surface. In contrast is the beautiful and surprising theorem of Widom [15] which states that the spectrum of a Toeplitz operator is connected.

One of the reasons that Toeplitz operator theory is not easy is that the equation $T_{\varphi \psi}=T_{\varphi} T_{\psi}$ does not hold in general (e.g. take $\varphi=e_{1}$ and $\psi=\bar{e}_{1}$ ). But there is a subclass of Toeplitz operators for which the above equality does hold: We say $T_{\psi}$ is analytic if $\psi \in H^{\infty}$. In this case we have $T_{\varphi} T_{\psi}=T_{\varphi \psi}$ and $T_{\bar{\psi}} T_{\varphi}=T_{\bar{\psi} \varphi}$ for all $\varphi \in L^{\infty}$. Hence the map

$$
H^{\infty} \rightarrow B\left(H^{2}\right), \quad \psi \mapsto T_{\psi},
$$


is an algebra isomorphism onto the closed subalgebra $A$ of all analytic Toeplitz operators. One can easily show that $A$ is the commutant of $U$, i.e. the set of all operators commuting with $U$, and so a maximal commutative subalgebra of $B\left(H^{2}\right)$. The open unit disc $\Delta$ can be embedded in the character space of the Banach algebra $H^{\infty}$ in a natural way, and if $\psi \in H^{\infty}$ then its Gelfand transform $\hat{\psi}$,restricted to $\Delta$ is a bounded analytic function. (By the way, the character space of $H^{\infty}$ is quite complicated. The famous Corona Theorem of Carleson says that $\Delta$ is dense in this space.) One can now state Wintner's theorem [16] : If $\psi \in H^{\infty}$ then $\sigma\left(T_{\psi}\right)=c l(\hat{\psi}(\Delta))$ where $c l$ denotes closure in C.

In the next section we shall indicate how one can use $\mathrm{C}^{*}$-algebras to get some other results of the classical theory of Toeplitz operators, but we end this section with a brief remark on Wiener-Hopf operators.

If $\varphi \in L^{1}(\mathbb{R})$ the Wiener-Hopf operator $W_{\varphi} \in B\left(L^{2}\left(\mathbb{R}^{+}\right)\right)$is defined by setting

$$
\left(W_{\varphi} f\right)(x)=\int_{0}^{\infty} \varphi(x-t) f(t) d t \quad\left(f \in L^{2}\left(\mathbb{R}^{+}\right)\right) .
$$

If $z \in \mathbb{C} \backslash\{0\}$ then the Wiener-Hopf equation is

$$
\left(z+W_{\varphi}\right) f=g
$$

where $g$ is given and $f$ is the unknown function. The conformal map of the upper half-plane onto the unit disc sets up a unitary equivalence between a Wiener-Hopf operator and its corresponding Toeplitz operator.

\section{$2 C^{*}$-algebras and Toeplitz operators}

For $T$ a bounded linear operator on a Hilbert space $H, N(T)$ denotes its aullspace $\{x \in H: T x=0\}$. Recall that $T$ is Fredholm if $T(H)$ is closed and $N(T), N\left(T^{*}\right)$ are finite-dimensional. In this case the Fredholm index is defined to be index $(T)=\operatorname{dim} N(T)-\operatorname{dim} N\left(T^{*}\right)$. The essential spectrum of $T$ is the set

$$
\sigma_{e}(T)=\{z \in \mathrm{C} \mid z 1-T \text { is not Fredholm }\} .
$$

Obviously $\sigma_{e}(T) \subseteq \sigma(T)$. There is a very useful characterization of Fredholm operators due to Atkinson, but to state it we need to introduce a few more concepts. An operator is compact if it is the norm limit of a sequence of finiterank operators. The set $K(H)$ of these operators on $H$ forms a closed ideal, and the quotient algebra $B(H) / K(H)$ is called the Colkin algebra. If $\pi$ is the canonical map from $B(H)$ to $B(H) / K(H)$ then the Atkinson characterization is that $T$ is Fredholm if and only if $\pi(T)$ is invertible in the Calkin algebra.

A key step in the spectral analysis of Toeplitz operators is the following lemma due to Coburn [2]. The proof is so short that we include it, as it also illustrates nicely the connections with Function Theory.

Lemma If $\varphi$ is a function in $L^{\infty}$ not almost everywhere equal to zero then either $T_{\varphi}$ or $T_{\varphi}^{*}$ has zero nullspace.

Proof Recall that the theorem of F. and M. Riesz says that a nonzero function $f$ in $H^{2}$ cannot vanish on any set of positive measure in $\mathrm{T}$. Now suppose that $f \in N\left(T_{\varphi}\right)$ and that $g \in N\left(T_{\varphi}^{*}\right)$. Then $\overline{\varphi f}$ and $\varphi \bar{g}$ are in $H^{2}$, so $\varphi f \bar{g}$ and $g \overline{\varphi f}$ are in $H^{1}$. By the "analyticity" property of the Hardy spaces, we must have $\varphi f \bar{g}$ is constant a.e. But $\int \varphi f \bar{g} d \lambda=0$, so $\varphi f \bar{g}=0$ a.e. If neither $f$ or $g$ is zero, then by the F. and M. Riesz theorem we must have $\varphi=0$ a.e., a contradiction. QED

It is immediate from this Iemma that if $T_{\varphi}$ is Fredholm then $T_{\varphi}$ is invertible if and only if index $\left(T_{\varphi}\right)=0$. From this it is not difficult to prove:

Theorem (Krein-Widom-Devinatz) If $\varphi$ is a continuous function on $\mathrm{T}$ then the operator $T_{\varphi}$ is Fredholm if and only if $\varphi$ does not vanish anywhere, and in this case index $\left(T_{\varphi}\right)$ is equal to minus the winding number of $\varphi$ with respect to the origin.

This beautiful result thus identifies an analytic index with a topological index, and is a simple prototype of the Atyiah-Singer Index Theorem. A direct consequence of it is the fact that the spectrum of $T_{\varphi}$ is connected if $\varphi$ is continuous, thus giving an easy proof of a special case of Widom's Theorem.

Many of the above results (and other results) are obtained by $C^{*}$-algebraic techniques. The idea is this: Let $T(Z)$ denote the $C^{*}$-algebra generated by the Toeplitz operators with continuous symbol (the reason for the appearance of the symbol $\mathbb{Z}$ will become clear presently). Then its commutator ideal (i.e. the smallest closed ideal $I$ for which the quotient algebra modulo $I$ is abelian) is $K\left(H^{2}\right)$. The map

$$
C(\mathbb{T}) \rightarrow T(\mathbb{Z}) / K\left(H^{2}\right), \varphi \mapsto T_{\varphi}+K\left(H^{2}\right),
$$


is a *-isomorphism. To see how this gives connectedness of the essential spectrum note that if $\varphi \in C(T)$ then its spectrum is just its range, so it is connected as $\mathrm{T}$ is connected. It follows that the spectrum of $T_{\varphi}+K\left(H^{2}\right)$ is connected, and by the Atkinson characterization this is just the essential spectrum of $T_{\varphi}$. With a little more work a simple proof that $\sigma\left(T_{\varphi}\right)$ has connected spectrum can be given in this case.

One can also consider the $C^{*}$-algebra generated by all Toeplitz operators, and use it to derive various results. For details see Douglas [4].

The above discussion indicates the usefulness of the algebra $T(Z)$ in Single Operator Theory, but it is also useful in $\mathrm{C}^{*}$-algebra theory. It is generated by a non-unitary isometry (viz $U$ ), and up to isomorphism it is the only such $C^{*}$-algebra. Moreover one can use the short exact sequence

$$
0 \rightarrow K\left(H^{2}\right) \rightarrow T(Z) \rightarrow C(\mathrm{~T}) \rightarrow 0
$$

(or rather a reduced form of it) to give a relatively easy proof of the Bott periodicity theorem in $\mathrm{K}$-theory (for locally compact spaces and for $\mathrm{C}^{*}$-algebras).

It is natural that one should try to extend these ideas and techniques to more general situations, and this has been done by many mathematicians including Douglas, Devinatz, Howe, Kaminker, Muhly and Singer and many others. We now discuss one of these extensions.

\section{$3 \quad$ Extended theories of Toeplitz operators}

An ordered group is a pair $(G, \leq)$ consisting of a (discrete) abelian group $G$ and a linear partial order $\leq$ on $G$ which is translation invariant (i.e. $x \leq y$ implies that $x+z \leq y+z$ ). Obvious examples are $\mathbb{Z}, \mathbb{R}$ and all subgroups of $\mathbb{R}$. Ordered groups exist in superabundance, for if $G$ is a discrete abelian group with Pontryagin dual group $\hat{G}$ then the following are equivalent conditions:

(1) There is a linear order $\leq$ on $G$ making $G$ an ordered group.

(2) $G$ is torsion-free

(3) $\hat{G}$ is connected.

Fix an ordered group $G$, and let $m$ denote normalized Haar measure on $\hat{G}$, and $L^{p}=L^{p}(\hat{G}, m), 1 \leq p \leq+\infty$. If $f \in L^{1}$ we say that $f$ is of analytic type if the Fourier transform $\hat{f}(x)=0$ for all $x \in G$ for which $x<0$. $H^{p}=H^{p}(G)$ denotes the norm-closed vector subspace of all $f \in L^{p}$ of analytic type. As is well known if $\varepsilon(x): \hat{G} \rightarrow T, \gamma \mapsto \gamma(x),(x \in G)$ then $(\varepsilon(x))_{x \in G}$ form an orthonormal basis for $L^{2}$. It follows that if $G^{+}=\{x \in G: 0 \leq x\}$ then $(\varepsilon(x))_{x \in G^{+}}$is an orthonormal basis for $H^{2}$. One can extend Fourier Analysis and Function Theory to this context (see Rudin [12]). The generalized Hardy spaces $H^{p}$ display analytic behaviour, for if $f, \bar{f} \in H^{p}$ then $f=$ constant a.e. We can now define Toeplitz operators as before, and many of the elementary results extend easily. However we shall primarily be interested in certain $\mathrm{C}^{*}$ algebras generated by Toeplitz operators.

Let $T(G)$ be the $C^{*}$-algebra generated by all $T_{\varphi}$ for which $\varphi \in C(\hat{G})$, and let $K(G)$ be its commutator ideal. Before stating some results in this area we need a few definitions.

A $C^{*}$-algebra $A$ is primitive if it has a faithful irreducible representation (i.e. there is an injective $*$-homomorphism $\varphi: A \rightarrow B(H)$ where $H$ is some Hilbert space with no nontrivial subspace invariant for every $\varphi(a)(a \in A))$. A is simple if it has no closed ideals apart from 0 and $A$. Simple $C^{*}$-algebras are primitive. In a loose sense the primitive and simple $C^{*}$-algebras are thought of as the building blocks from which all $\mathrm{C}^{*}$-algebras are made, and for this and other reasons it is very important to have many examples of such algebras.

Theorem Let $G$ be an ordered group.

(1) $T(G)$ is primitive (and therefore $K(G)$ is primitive also).

(2) $K(G)$ is simple if and only if $G$ is (order isomorphic to) a subgroup of $\mathbb{R}$.

(1) and the forward implication in (2) are due to the author [9]. The backward implication in (2) is due to Douglas [5]. The study of the algebras $K(G)$ in the case of subgroups of $\mathbf{R}$ has become especially important recently with connections having been discovered with Connes' non- commutative Differential Geometry. There are are many more interesting things that can be said about these more general Toeplitz theories. For example there is the very rich spatial theory due to Muhly and others which has not even been touched on above. We finish up with a few remarks on the $\mathrm{K}$-theory of the algebras $K(G)$. In [5] Douglas asked if subgroups $G_{1}, G_{2}$ of $\mathbb{R}$ were order isomorphic when $K\left(G_{1}\right)$ and $K\left(G_{2}\right)$ are isomorphic. This was answered affirmatively in [10] in a special case, and in general in [7]. The method of proof in both cases involved computing the K-groups of $K(G)$.

A good elementary introduction to the theory of Toeplitz operators is [4]. 


\section{References}

[1] A. Brown and P. R. Halmos, Algebraic properties of Toeplitz operators, J. Reine Angew. Math. 231 (1963), 89-102.

[2] L. A. Coburn, Weyl's theorem for non-normal operators, Michigan Math. J. 13 (1966), 285-286.

[3] A. Devinatz, Toeplitz operators on $H^{2}$-spaces, Trans. Amer. Math. Soc. 112 (1964), 304-317.

[4] R. G. Douglas, Banach Algebra Techniques in Operator Theory, Academic Press, New York and London, 1972.

[5] R. G. Douglas, On the $C^{*}$-algebra of a one-parameter semigroup of isometries, Acta Math. 128 (1972), 143-152.

[6] P. Hartman and A. Wintner, The spectra of Toeplitz's matrices, Amer. J. Math. 76 (1954), 867-882.

[7] R. Ji and J. Xia, On the classification of commutator ideals, J. Funct. Anal. (to appear).

[8] M. G. KreIn, Integral equations on a half-line with kernel depending on the difference of the arguments, Uspehi Mat. Nauk 13 (1958), no. 5 (83), 3-120.

[9] G. J. Murphy, Ordered groups and Toeplitz algebrus, J. Operator Theory 18 (1987), 303-326.

[10] G. J. Murphy, Simple $C^{*}$-algebras and subgroups of Q, Proc. Amer. Math. Soc. (to appear).

[11] M. Rosenblum, A concrete spectral theory of self-adjoint Toeplitz operators, Amer J. Math. 87 (1965), 709-718.

[12] W. Rudin, Fourier Analysis on Groups, Interscience Publishers, New York and London, 1962.

[1] 3] O. Toeplitz, Zur theorie der quadratischen Formen von unendlichvielen Veranderlichen, Math. Ann. 70 (1911), 351-376.
[14] H. Widom, Inversion of Toeplitz matrices II, Illinois J. Math. 4 (1960), 88-99.

[15] H. Widom, On the spectrum of a Toeplitz operator, Pacific J. Math. 14 (1964), 365-375.

[16] A. Wintner, Zur theorie der beschränkten Bilinear formen, Math. Z. 30 (1929), 228-282.

Department of Mathematics

University College Cork 